\title{
Deformaciones en áreas volcánicas: una aproximación numérica para su predicción en el volcán Teide (Tenerife, Islas Canarias)
}

\author{
M. ChARCO ${ }^{1} \&$ P. GALÁN DEL SASTRE ${ }^{2}$ \\ ${ }^{1}$ Instituto de Geociencias (CSIC-UCM) \\ mcharco@iag.csic.es \\ ${ }^{2}$ Departamento de Matemática Aplicada a la Edificación, al Medio Ambiente y al Urbanismo, ETS \\ Arquitectura, UPM. \\ pedro.galan@upm.es
}

Recibido: 3/09/2011

Aceptado: 5/10/2011

\begin{abstract}
Resumen
El estudio de áreas volcánicas activas depende tanto de la disponibilidad de observaciones físicas de los cambios que se producen en el medio natural como de la interpretación de estos cambios. Así, hoy en día, el desarrollo y aplicación de técnicas geodésicas espaciales, como el GPS (Global Positioning System) o el InSAR (Interferometry with Synthetic Aperture Radar), proporciona una visión sin precedentes de las deformaciones en zonas volcánicas. Estas deformaciones son el reflejo de distintos procesos de origen tectónico, magmático e hidrotermal, de difícil observación y registro, que se producen en el interior del medio. En este sentido, el desarrollo de modelos para la predicción de deformaciones permite establecer un enlace directo entre los procesos observados en superficie y los que se producen en profundidad, lo que podría resultar crucial para la evaluación de riesgos volcánicos. En este trabajo, nos planteamos el desarrollo de un modelo físico para estudiar las deformaciones elásticas consecuencia de una variación de presión en el medio. Este modelo lo hemos implementado numéricamente mediante el Método de Elementos Finitos (FEM). La utilización del FEM permite considerar distintas características estructurales y morfológicas del medio así como sus heterogeneidades mecánicas. La simulación de deformaciones en Tenerife (Islas Canarias), considerando diferentes hipótesis sobre el medio, nos permite concluir que las predicciones de un modelo pueden describir de forma muy precisa cualquier conjunto de datos observacionales. Sin embargo, la exactitud de estas predicciones va a depender de las hipótesis realizadas sobre el medio.
\end{abstract}

Palabras clave: Modelización, Método de Elementos Finitos, Desplazamientos, Tenerife.

\section{Deformation in volcanic areas: a numerical approach for their prediction in Teide volcano (Tenerife, Canary Islands)}

\begin{abstract}
Active volcanic areas study comprises both, observation of physical changes in the natural media and the interpretation of such changes. Nowadays, the application of spatial geodetic techniques, such as GPS (Global Positioning System) or InSAR (Interferometry with Synthetic Aperture Radar), for deformation understanding in volcanic areas, revolutionizes our view of this geodetic signals. Deformation of the Earth's surface reflects tectonic, magmatic and hydrothermal processes at depth. In this way, the prediction of volcanic deformation through physical modelling provides a link between the observation and depth interior processes that could be crucial for volcanic hazards assessment. In this work, we develop a numerical model for elastic deformation study. The Finite Element Method (FEM) is used for the implementation of the numerical model. FEM allows to take into account different mor-
\end{abstract}


phology, structural characteristics and the mechanical heterogeneities of the medium. Numerical simulations of deformation in Tenerife (Canary Islands) taking into account different medium hypothesis allow us to conclude that the accuracy of the predictions depends on how well the natural system is described.

Keywords: Modelling, Finite Element Method, Displacement field, Tenerife.

\section{Referencia normalizada}

Charco, M., Galán del Sastre, P., (2011). Deformaciones en áreas volcánicas: una aproximación numérica para su predicción en el volcán Teide (Tenerife, Islas Canarias). Física de la Tierra Vol. 23 Núm. 1 (2011): 83-92

Sumario: Introducción. 1. Metodología. 2. Modelo numérico del Teide (Tenerife, Islas Canarias). 3. Discusión y Conclusiones. 4. Agradecimientos. Referencias bibliográficas.

\section{Introducción}

En los últimos años, el avance tanto de la precisión y resolución espacio-temporal de técnicas geodésicas espaciales, como el GPS o el InSAR (Interferometría con Radar de Apertura Sintética), como de la modelización cuantitativa de deformaciones ha permitido profundizar en el estudio de las deformaciones de la corteza terrestre en áreas volcánicas con el fin de estimar la localización, geometría y variaciones de volumen de distintos cuerpos magmáticos (p.e., Dzurisin, 2003). Sin embargo, y a pesar de los avances técnicos y computacionales, el modelo más utilizado, gracias a su simplicidad, sigue siendo el modelo de Mogi (Mogi, 1958). Este modelo, bajo la hipótesis de que el medio es un semiespacio elástico, isótropo y homogéneo, se utiliza para describir el campo de deformaciones producido por la presurización de una cavidad esférica cuyo radio es menor que su profundidad. No obstante, este tipo de simplificaciones puede causar errores a la hora de interpretar deformaciones en términos de estimación de características de fuente ya que, generalmente, los volcanes son mecánicamente heterogéneos y presentan estratificaciones que afectan a la propagación del magma, al campo de esfuerzos y, por tanto, a la deformación en superficie (p.e., Gudmundsson, 2006). Por ejemplo, Bonafede y Ferrari (2009) demuestran que la estimación de parámetros como la profundidad y la localización dependen de la reología del medio (elástica o viscoelástica) levemente mientras que otros parámetros como el incremento/descenso de presión sobre la presión litostática del medio depende fuertemente de la reología. Por tanto, parece claro que la interpretación de deformaciones en áreas volcánicas debería tener en cuenta una descripción realista del medio en el que se encuentra la fuente. El Método de Elementos Finitos (también conocido por su acrónimo inglés -FEM) permite considerar geometrías complicadas así como distintas reologías, geometrías de fuente, características estructurales, etc., $\mathrm{y}$, por tanto, al utilizar este método se podrían corregir distintas desviaciones de los parámetros de fuente obtenidas a partir de modelos, como el de Mogi, con respecto a sus valores reales.

La interpretación de deformaciones mediante el Método de Elementos Finitos comenzó en la época de los 70 con el trabajo de Dieterich y Decker (1975) en el que se estudia el efecto de fuentes de diferentes geometrías llegándose a la conclusión 
de que, para distinguir la geometría de una fuente determinada, es necesario considerar las componentes horizontales del vector desplazamiento ya que el patrón de los desplazamientos verticales es independiente de esta geometría. Entre los trabajos más recientes se encuentran, por citar algunos, los de Newman et al. (2001, 2006) que utiliza esta metodología numérica para estudiar el efecto de la viscoelasticidad de los materiales que rodean una posible intrusión de forma elipsoidal; el de Masterlark (2007) en el que se consideran una serie de configuraciones con el fin de estudiar los efectos de las hipótesis del modelo de Mogi sobre la interpretación de deformaciones en la caldera de Okmok (EE. UU.); y, finalmente, el de Manconi et al. (2010) que estudia los efectos de la estratificación mecánica de las heterogeneidades del medio en los Campos Flegreos (Italia).

El objeto de este trabajo es proporcionar una herramienta numérica para simular las deformaciones que causaría una intrusión bajo el volcán Teide (Islas Canarias). Dado que en este volcán el relieve del terreno es significativo, además de estudiar el efecto de las heterogeneidades mecánicas del medio consideramos su topografía. Para ello, utilizaremos FEM ya que es un método robusto y preciso en problemas que consideran geometrías complejas y que precisan de mallas irregulares.

\section{Metodología}

La hipótesis de partida clave para la modelización de deformaciones en áreas volcánicas es que estas deformaciones son el reflejo en superficie de procesos de origen tectónico, magmático y/o hidrotermal que se producen en profundidad. Estos procesos generan una variación de esfuerzos que se transmiten en el medio como consecuencia de sus propiedades mecánicas. Hoy en día, su estudio se realiza desde el punto de vista elasto-estático suponiendo que las deformaciones no varían con el tiempo entendiendo éste como el dado por el muestreo temporal de las técnicas geodésicas utilizadas para el registro de desplazamientos. Así, el modelo utilizado para la predicción de deformaciones en un sólido elástico, $\Omega$, viene dado por:

$$
\begin{array}{rrr}
\nabla \cdot \boldsymbol{\sigma}+\rho \mathbf{f}=0 & \text { en } \quad \Omega \\
\sigma_{\mathrm{ij}}=\lambda \sum_{k=1}^{3} \varepsilon_{k k} \delta_{i j}+2 \mu \varepsilon_{i j} & \text { en } \quad \Omega \\
\boldsymbol{\sigma} \cdot \mathbf{n}=0 & \text { en } \quad \Gamma_{1} \\
\mathbf{u}=0 & \text { en } \quad \Gamma_{2},
\end{array}
$$

donde, en la primera expresión del problema (1), $\sigma$ representa el tensor de esfuer$z o s, \varepsilon$, es el tensor de deformaciones y $\rho \mathbf{f}$ son las fuerzas de cuerpo por unidad de volumen (siendo $\rho$ la densidad del sólido), es decir, el término fuente de la ecuación. La segunda expresión de (1) se corresponde con la relación entre las deformaciones y los esfuerzos, es decir, la ley constitutiva del medio en la $\lambda$ y $\mu$ son los parámetros de Lamé, que serán variables y dependientes de la posición en el medio, y $\delta_{\mathrm{ij}}$ es la delta de Kronecker. Las condiciones de contorno reflejan que la frontera 
superior del medio $\left(\Gamma_{1}\right)$ se encuentra libre de esfuerzos mientras que en las fronteras laterales e inferior $\left(\Gamma_{2}\right)$ los desplazamientos son nulos. Generalmente, se considera que los esfuerzos se producen por una variación de presión en el medio uniforme lo que se simula mediante tres dipolos de fuerza mutuamente ortogonales (centro de dilatación). Para representar matemáticamente este tipo de términos fuente en la primera expresión del problema (1) consideramos una función gaussiana, aprovechando la propiedad de que cuando la varianza tiende a cero este tipo de funciones convergen a la delta de Dirac:

$$
\rho f=a^{3} \Delta P \frac{\lambda+2 \mu}{\mu} \pi \frac{1}{\sigma_{x_{1}} \sigma_{x_{2}} \sigma_{x_{3}} \pi^{3 / 2}} \nabla\left[e^{-\left(\frac{\left(x_{1}-x_{1}^{\prime}\right)^{2}}{\sigma_{x_{1}}}+\frac{\left(x_{2}-x_{2}^{\prime}\right)^{2}}{\sigma_{x_{2}}}+\frac{\left(x_{3}-x_{3}^{\prime}\right)^{2}}{\sigma_{x_{3}}}\right)}\right]
$$

donde $\sigma_{x i}$ es la varianza de la función gaussiana en la dirección $x_{i}$. Cuando $\sigma_{x i}=$ $a$, esta función representa una fuente esférica de radio $a . \Delta \mathrm{P}$, dependiendo de su signo, corresponde al incremento/descenso de presión sobre la presión litostática del medio.

Las simulaciones numéricas para los distintos casos considerados en este trabajo se realizarán mediante FEM. Este método se encuentra descrito ampliamente en la trabajos como el de Brenner y Scott (2008).

\section{Modelo numérico del Teide (Tenerife, Islas Canarias)}

El complejo Teide-Pico Viejo, activo hoy en día, domina el sistema eruptivo de la isla más grande del Archipiélago Canario, Tenerife (Ablay et al., 1995; 1998). Estos dos estratovolcanes se solapan formando un edificio doble cuya máxima altitud corresponde al pico del Teide ( $3718 \mathrm{~m}$ sobre el nivel del mar). Aunque las erupciones en el sistema son mayoritariamente efusivas, también se han encontrado indicios de erupciones explosivas en el pasado. La última erupción explosiva se produjo hace 2000 años en Montaña Blanca que se encuentra situada en uno de los flancos del Teide. Martí et al. (2008) señalan que la evolución magmática del sistema podría continuar con una tendencia a producir un volumen mayor de magmas fonolíticos lo que incrementaría la posibilidad de que, en el futuro, se produjeran erupciones explosivas. En este trabajo consideramos la posibilidad de una erupción en las cercanías de este centro de emisión teniendo en cuenta que una intrusión de magma previa a ésta podría causar deformaciones del terreno. Así, las simulaciones numéricas llevadas a cabo mediante FEM tienen por objeto estimar la deformación en la isla de Tenerife causada por una presurización hipotética de un reservorio magmático somero situado bajo la cima del volcán Teide.

Para esta aplicación, vamos a tener en cuenta tanto la topografía del terreno como las heterogeneidades del medio. El dominio tridimensional se trata de un volumen de $200 \times 200 \times 100 \mathrm{~km}^{3}$ (Figura 1) en las direcciones $x_{1}(\mathrm{OE}), x_{2}(\mathrm{SN})$ y $x_{3}($ positivo hacia el exterior del dominio) que cubre por completo la isla de Tenerife. El 
sistema de coordenadas cartesianas se encuentra centrado bajo la cima del Teide al nivel del mar. La discretización del dominio consta de 248155 hexaedros y 253500 nodos para cada una de las componentes del vector desplazamiento. Esta discretización es más fina en la zona en la que se localiza la fuente y tiene en cuenta las principales características estructurales de la isla. La frontera del dominio correspondiente a la superficie del terreno se ha construido a partir de un modelo digital del terreno del Centro Nacional de Información Geográfica (CNIG) y de un modelo batimétrico (Smith and Snadwell, 1997). Esta frontera se encuentra libre de esfuerzos normales. En el resto de fronteras que delimitan el dominio se considera que los desplazamientos son cero. La fuente con una intensidad, $f_{0}$, de $10 \mathrm{MPa} \mathrm{km}$, se encuentra situada en el punto de coordenadas $(0,0,-5) \mathrm{km}$, es decir, a $5 \mathrm{~km}$ de profundidad bajo la cima del Teide. Para la localización de la fuente se han tenido en cuenta estudios petrológicos (Ablay, 1998; Andújar et al., 2010).

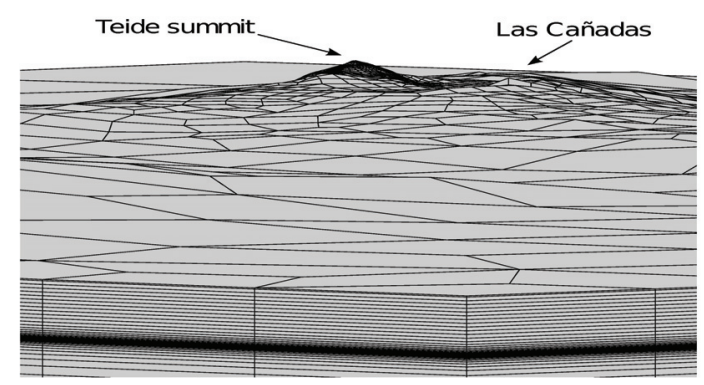

Fig. 1. Detalle del mallado tridimensional del dominio de cálculo. Entorno al área de localización de la fuente su resolución es de $50 \mathrm{~m}$, aumentando al alejarse de la fuente.

Un aspecto importante de las simulaciones realizadas se refiere a las propiedades mecánicas del medio. En la Figura 2 mostramos un perfil E-O del modelo tridimensional de parámetros elásticos. Las propiedades mecánicas del medio se han obtenido a partir de estudios sísmicos (Watts et al., 1997) y gravimétricos (Camacho et al., 2011) considerando la relación empírica de Nafe-Drake (Nafe \& Drake, 1963) y el modelo PREM (Dziewonski \& Anderson, 1981). Mediante este modelo de heterogeneidades consideramos tanto variaciones en profundidad, que son las que habitualmente se tienen en cuenta (p.e., Fernández et al., 2009) como variaciones laterales de los parámetros elásticos. 


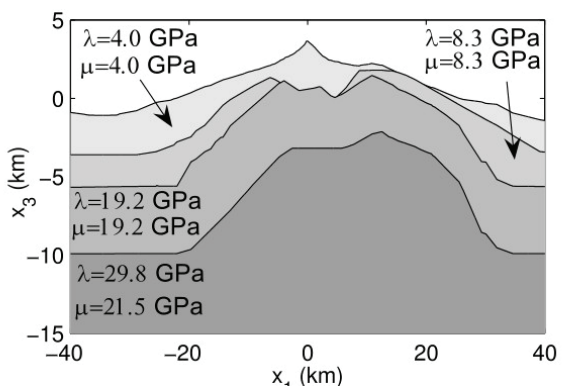

Fig. 2. Perfil vertical en $\mathrm{x}_{2}=0$ del modelo tridimensional de parámetros elásticos de la isla de Tenerife

Los resultados de las simulaciones realizadas se muestran en la Figura 3. El área deformada se restringe a las inmediaciones del volcán siendo estas deformaciones indistinguibles del ruido a distancias superiores a $10 \mathrm{~km}$ desde la cima del volcán. El desplazamiento vertical alcanza una magnitud máxima de aproximadamente 9 $\mathrm{cm}$. Este máximo se alcanza en las inmediaciones de la cima del volcán, en la que aparece un mínimo relativo debido al efecto de la topografía del terreno. Por tanto, teniendo en cuenta la precisión que se alcanza hoy en día con técnicas geodésicas espaciales como el GPS en continuo $(0.2-0.6 \mathrm{~cm}$ y $0.5-1 \mathrm{~cm}$ en la horizontal y vertical, respectivamente - Dixon et al., 1997; Bartel et al., 2003) o en la realización de campañas GPS periódicas (1-3 cm -Segall and Davis, 1997), los desplazamientos resultado de considerar una intrusión de magma en un reservorio somero bajo la cima del Teide y su consiguiente cambio de presión serían detectables mediante estas técnicas observacionales. Considerando la dependencia lineal de la magnitud del desplazamiento con respecto a la variación de presión del reservorio y las precisiones mencionadas, estas técnicas podrían detectar una variación de presión de, aproximadamente, $3.5 \mathrm{MPa} \mathrm{km}$ bajo la cima del volcán.

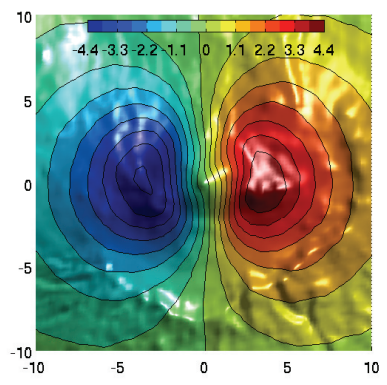

(a)

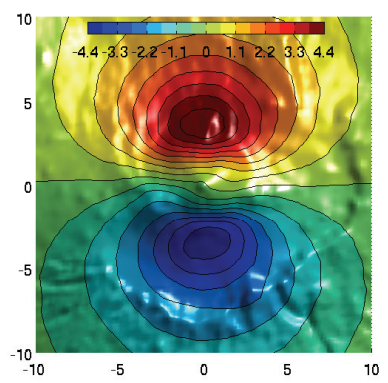

(b)

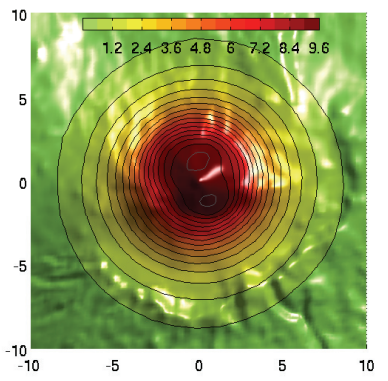

(c)

Fig. 3. Campo de desplazamientos verticales causados por una fuente esférica localizada a $5 \mathrm{~km}$ de profundidad bajo la cima del volcán Teide en un medio homogéneo cuya frontera superior se sitúa a una altura de $x_{3}=3718 \mathrm{~m}$ (altitud del Teide). (a) $u_{1}$, (b) $u_{2}$ y (c) $u_{3}$. 


\section{Discusión y Conclusiones}

En este trabajo hemos desarrollado un modelo numérico tridimensional de la isla de Tenerife que considera una distribución realista de las heterogeneidades verticales y laterales del medio así como de su topografía. Este modelo permite predecir e interpretar las deformaciones que causaría una variación de presión bajo la cima del volcán Teide. Estas variaciones de presión normalmente se producen como consecuencia de la intrusión de material desde niveles más profundos a reservorios más someros y/o a la variación del contenido de volátiles y/o gases en estos reservorios, fenómenos ambos que podrían desencadenar una erupción. Por tanto, teniendo en cuenta que estos efectos se podrían encontrar entre los precursores de una erupción, la metodología propuesta podría ayudar al desarrollo y mejora de sistemas de vigilancia geodésicos en Tenerife y, por tanto, al desarrollo de sistemas de alerta frente a riesgos volcánicos asociados a una posible erupción.

Normalmente, la interpretación de deformaciones en zonas volcánicas se realiza mediante el modelo de Mogi (Mogi, 1958), que permite obtener predicciones muy precisas del vector desplazamiento en una zona determinada. Este modelo, en el que se considera la Tierra como un semiespacio elástico, isótropo y homogéneo, permite obtener soluciones analíticas cerradas y simples del problema (1), por lo que es el preferido a la hora de la interpretación de deformaciones. En la Figura 4 observamos los resultados de las simulaciones obtenidas al considerar un dominio tridimensional de características similares al considerado mediante el modelo de Mogi. La superficie libre del medio, en este caso plana, la hemos localizado en $x_{3}$ igual a la altura del Teide sobre el nivel del mar. El medio es isótropo y homogéneo, es decir, los parámetros elásticos son constantes en el medio. En este caso $\lambda=\mu=19.2 \mathrm{GPa}$, siendo éste el valor correspondiente al estrato en el que se localiza la intrusión. Comparando las Figuras 3 y 4 observamos discrepancias notables debidas al efecto de las hipótesis de los dos modelos. La topografía local del complejo Teide-Pico Viejo y las variaciones laterales y en profundidad de los parámetros elásticos producen variaciones de patrón con respecto a los desplazamientos obtenidos mediante el modelo de Mogi. Estas diferencias de patrón se observan tanto en los desplazamientos horizontales como en los verticales, que con el modelo de Mogi alcanzan su máximo valor en la proyección de la fuente en superficie, es decir, donde se localizaría la cima del Teide. Además de estas diferencias de patrón también se producen diferencias en la magnitud de los desplazamientos. Por ejemplo, el desplazamiento vertical máximo es de aproximadamente $1.5 \mathrm{~cm}$ para el caso del medio homogéneo (Figura 4) mientras que con el modelo numérico del Teide este valor es de aproximadamente $9 \mathrm{~cm}$ (Figura 3). Estas diferencias se deben a que la falta de cohesión de los materiales situados sobre los estratos superiores al estrato en que se encuentra la intrusión proporciona valores de $\mu$ pequeños, que amplifican la magnitud del desplazamiento.

Como consecuencia de las discrepancias señaladas podemos concluir que la utilización del Modelo de Mogi para la interpretación de deformaciones en zonas volcánicas, en términos de estimación de parámetros que caracterizan la posible fuente de deformación, puede conducir a errores de estimación. Así, la fiabilidad de las 
interpretaciones de la deformación observada en áreas volcánicas activas, basadas en los resultados obtenidos a partir de las simulaciones numéricas de un modelo físico, es una cuestión de precisión vs. exactitud. Como hemos señalado anteriormente, las predicciones obtenidas mediante el modelo de Mogi pueden describir de forma muy precisa un determinado conjunto de datos observacionales, sin embargo, la exactitud de estas predicciones dependerá de lo bien que el modelo represente el medio en el que se localiza la variación de presión. En este sentido el modelo tridimensional de Tenerife implementado mediante FEM permite considerar características como la topografía o las heterogeneidades del medio que no se han tenido en cuenta mediante otros modelos más simples desde el punto de vista físicomatemático como el modelo de Mogi.

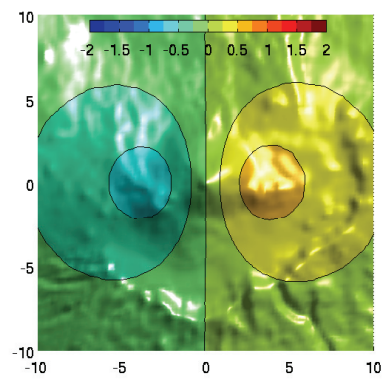

(a)

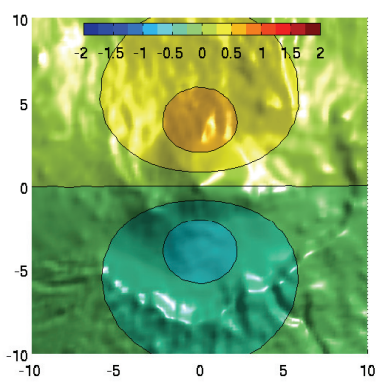

(b)

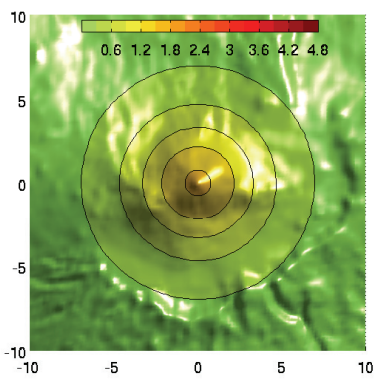

(c)

Fig. 4. Campo de desplazamientos verticales causados por una fuente esférica localizada a $5 \mathrm{~km}$ de profundidad bajo la cima del volcán Teide en un medio homogéneo cuya frontera superior se sitúa a una altura de $x_{3}=3718 \mathrm{~m}$ (altitud del Teide). (a) $u_{1}$, (b) $u_{2}$ y (c) $u_{3}$.

\section{Agradecimientos}

Este trabajo ha sido financiado por el proyecto CSIC-MICINN con referencia 2009301053.

\section{Referencias bibliográficas}

ABLAY, G.J., ERNST, G.G.J., MARTÍ, J. \& SPARKS, R.S.J. (1995). The 2ka subplinian eruption of Montaña Blanca, Tenerife. Bull Volcanol 57, 337-355.

doi: $10.1007 / \mathrm{BF} 00301292$

http://dx.doi.org/10.1007/BF00301292

ABLAY, G.J., CARROLL, M.R., PALMER, M.R., MARTÍ, J. SPARKS, R.S.J. (1998).

Basanite-phonolite lineages of Teide-Pico Viejo volcanic complex, Tenerife, Canary Islands, J. Petrol. 39(5), 905-936.

doi: $10.1093 /$ petroj/39.5.905

http://dx.doi.org/10.1093/petroj/39.5.905 
ANDÚJAR, J., COSTA, F., \& MARTÍ, J. (2010). Magma storage conditions of the last eruption of Teide volcano (Canary Islands, Spain). Bull. Volcanol. 172(4), 381-395.

doi: $10.1007 / \mathrm{s} 00445-009-0325-3$

http://dx.doi.org/10.1007/s00445-009-0325-3

BARTEL, B.A., HAMBURGER, M.W., MERTENS, C.M., LOWRY, A.R. \& CORPUZ, E. (2003). Dynamics of active magmatic and hydrothermal systems at Taal Volcano, Philippines, from continuous GPS measurements. J. Geophys. Res. 108(B10), 2475.

doi:10.1029/2002JB002194

http://dx.doi.org/10.1029/2002JB002194

BRENNER, S. \& SCOTT, R. (2008). The mathematical theory of Finite Element Methods.

Text in Applied Mathematics, 15, 402 p. Ed.: Springer.

doi: 10.1007/978-0-387-75934-0

http://dx.doi.org/10.1007/978-0-387-75934-0

CAMACHO, A.G., FERNÁNDEZ, J. \& GOTTSMANN, J. (2011). A new gravity inversion method for multiple subhorizontal discontinuity interfaces and shallow basins. J. Geophys. Res. 116, B02413.

doi: 10.1029/2010JB008023

http://dx.doi.org/10.1029/2010JB008023

DIETERICH, J.H. \& DECKER, R.W. (1975). Finite element modeling of surface deformation associated with volcanism. J. Geophys. Res. 80, 4094-4102.

doi: 10.1029/JB080i029p04094

http://dx.doi.org/10.1029/JB080i029p04094

DIXON, T., MAO, A., BURSIK, M., HEFLIN, M., LANGBEIN, J., STEIN, R. \& WEBB, F. (1997). Continuous monitoring of surface deformation at Long Valley Caldera, California, with GPS. J. Geophys. Res. 102, 12017-12034.

doi: 10.1029/96JB03902

http://dx.doi.org/10.1029/96JB03902

DZIEWONSKI, A.M. \& ANDERSON, D.L. (1981). Preliminary Reference Earth Model. Phys. Earth Planet. Int. 25, 297-356.

doi: 10.1016/0031-9201(81)90046-7

http://dx.doi.org/10.1016/0031-9201(81)90046-7

DZURISIN, D. (2003). A comprehensive approach to monitoring volcano deformation as a window on the eruption cycle. Rev. Geophys. 35, 343-384.

doi: 10.1029/2001RG000107

http://dx.doi.org/10.1029/2001RG000107

FERNÁNDEZ, J., TIZZANI, P., MANZO, M., BORGIA, A., GONZÁLEZ, P.J., MARTÍ, J., PEPE, A., CAMACHO, A.G., CASU, F., BERARDINO, P., PRIETO, J.F. \& LANARI, R. (2009). Gravity driven deformation of Tenerife measured by InSAR time series analysis. Geophys. Res. Lett. 36, L04306.

doi: 10.1029/2008GL036920

http://dx.doi.org/10.1029/2008GL036920

GUDMUNDSSON, A. (2006). How local stresses control magma-chamber rupture, dyke injections, and eruptions in composite volcanoes. Earth-Sci. Rev. 79(1-2), 1-31.

doi: 10.1016/j.earscirev.2006.06.006 
http://dx.doi.org/10.1016/j.earscirev.2006.06.006

MANCONI, A., WALTER, T.R., MANZO, M., ZENI, G., TIZZANI, P., SANSOTI, E. \& LANARI, R. (2010). On the effects of 3-D mechanical heterogeneities at Campi Flegrei caldera, southern Italy. J. Geophys. Res. 115, B08405.

doi: 10.1029/2009JB007099

http://dx.doi.org/10.1029/2009JB007099

MARTÍ, J., GEYER, A., ANDÚJAR, J., TEIXIDÓ, F. \& COSTA, F. (2008). Assessing the potential for future explosive activity from Teide-Pico Viejo stratovolcanoes (Tenerife, Islas Canarias). J. Volcanol. Geotherm. Res. 178, 529-542.

doi: 10.1016/jvolgeores.2008.07.011

http://dx.doi.org/10.1016/jvolgeores.2008.07.011

MASTERLARK, T. (2007). Magma intrusion and deformation predictions: Sensitivities to the Mogi assumptions. J. Geophys. Res. 112, B06419.

doi: 10.1029/2006JB004860

http://dx.doi.org/.1029/2006JB004860

MOGI, K. (1958). Relations of eruptions of various volcanoes and deformation around them. Bull. Earthquake Res. Inst. Univ. Tokyo 36, 99-134.

NAFE, J.E. \& DRAKE, C.L. (1963). Physical properties of marine sediments. In: Hill N. (Ed.) The Sea 3, Interescience, New York, 794-815.

NEWMAN, A.V., DIXON, T.H., OFOEGBU, G.I. \& DIXON, J.E. (2001). Geodetic and seismic constraints on recent activity at Long Valley Caldera, California: Evidence for viscoelastic rheology. J. Volcanol Geotherm. Res. 105, 183-206.

doi: 10.1016/S0377-0273(00)00255-9

http://dx.doi.org/10.1016/S0377-0273(00)00255-9

SEGALL, P. \& DAVIS, J. (1997). GPS applications for geodynamics and erthquake studies. Annu. Rev. Earth Planet. Sci. Lett. 25, 301-336.

doi: 10.1146/annurev.earth.25.1.301

http://dx.doi.org/10.1146/annurev.earth.25.1.301

SMITH, W.H.F. \& SANDWELL, D.T. (1997). Global sea floor topography from satellite altimetry and ship depth soundings, Science 277, 1956-1952.

doi: 10.1126/science.277.5334.1956

http://dx.doi.org/10.1126/science.277.5334.1956

WATTS, A.B., PEIRE, C., COLLIER, J. DALWOOD, R., CANALES, J.P. \& HENSTOCK, T.J. (1997). A seismic study of lithospheric flexure in the vicinity of Tenerife, Canary Islands. Earth Planet. Sci. Lett. 146(3-4), 431-447.

doi: 10.1016/S0012-821X(96)00249-X

http://dx.doi.org/10.1016/S0012-821X(96)00249-X 